\title{
BUSINESS PROCESS BASED INTEGRATION OF DYNAMIC COLLABORATIVE ORGANIZATIONS
}

\author{
Guillermo Jimenez, Manuel Ocampo, \\ Nathalie Galeano, Arturo Molina \\ Instituto Tecnologico y de Estudios Superiores de Monterrey, Mexico, \\ \{guillermo.jimenez,A00777498,ngaleano,armolina\}@itesm.mx
}

\begin{abstract}
$A$ virtual breeding environment (VBE) is an association of enterprises willing to collaborate in a network to provide goods or services, described by business processes. Two major components of the VBE are an e-catalogue of competences and a repository of business process templates specifying the necessary roles that enterprises should play to participate in collaboration networks for providing a specific service. Process templates should be concretised for particular needs and enterprise capabilities. Which enterprises shall participate in and the committed resources have to be decided at moment of template concretisation: once identified the process template for a business opportunity different enterprises could collaborate. Specific responsibilities are assigned to enterprises using information from semantically defined capabilities stored in the VBE's e-catalogue. This paper describes an approach for template process definition and concretisation in a simple way; thus enterprises could be assigned to purticipate in a business process when their capabilities and availability of resources are found to be the most appropriate. The capabilities of a tool implemented to perform process -template concretization is described.
\end{abstract}

\section{INTRODUCTION}

A dynamic virtual organization is characterised by a limited lifetime, and the flexibility of individual participants to join or leave the group during its lifetime (Chadwick, 2004). Virtual Breeding Environment (VBE) could help the creation, operation and dissolution of organizations in dynamic environments (ECOLEAD D21.1, 2005). A VBE represents a pool of organizations and their supporting institutions that have both the potential and the will to cooperate with each other through the establishment of a long-term cooperation agreement. When a business opportunity is identified by one member (acting as a broker); a subset of these organizations can be selected, thus forming a dynamic Virtual Organization (VO). In this way, VOs comprise sets of independent organizations that share resources and skills to achieve their goals (Camarinha-Matos, 2004).

The VBE is a leading concept for the "European Collaborative networked Organisations LEADership initiative" (ECOLEAD), a project funded by the 
European Commission under Sixth Framework Programme (www.ecolead.org). ECOLEAD's vision is that "In ten years, in response to fast changing market conditions, most enterprises and specially the SMEs will be part of some sustainable collaborative network that will act as breeding environment for the formation of dynamic virtual organizations".

The VBE contains a Profiling and Competency Management System (PCMS) which is an e-catalogue of enterprises' profiles and competencies willing to collaborate in virtual organizations. When a business opportunity arises and a VO needs to be created, potential VO members are found using the PCMS (ECOLEAD D21.2a, 2005).

A VO is characterised by a particular set of processes that need to be performed to fulfil the VO's goals. Business processes are stored in the VBE as process templates specifying necessary competences that need to be concretised. These business processes define in an abstract way the business logic needed for collaboration among enterprises, but no participants are included in the process definition. Once concretised the business processes will be used to orchestrate the information exchange among enterprises' and their supporting applications. In order to distinguish between a template and an executable business processes the first one is called a Generic Business Process (GBP) and the second one is called a specific business process a (SBP).

This paper describes an approach and associated tools for the definition of generic business processes and their concretisation into executable business processes, necessary to construct VOs specific to collaboration requirements as demanded by a business opportunity. The roles defined in GBPs are assigned to specific enterprises and their applications linked using Web Services (Hass, 2004) for interoperation among VO participants.

\section{VIRTUAL BREEDING ENVIRONMENTS AND VIRTUAL ORGANIZATIONS}

It is far less costly and much more effective to quickly build a VO in a breeding environment context than through a generalized partners' search. In other words, VBEs substantially contribute to increase the level of preparedness of their members for participation in potential collaborative processes. Clearly, the organizations need invest in getting prepared for collaboration within the VBE; therefore in order to make this investment worthwhile, the participation of organizations in the VBE must be beneficial. With the provided support through the VBE, it is foreseen that the VOs of the future can go far beyond the buy-sell activities, what is the state of the art for the current VOs (ECOLEAD 21.1, 2005). In ECOLEAD's concept, Virtual Organizations are networks of independent, dynamic and geographically dispersed traditional organizations with common goals.

A critical VBE component for VO creation and operation is the Profiling and Competency Management System (PCMS); it is a repository of organizations' profiles and competencies willing to collaborate in virtual organizations. When a business opportunity arises and a VO needs to be created, potential VO members are found using the PCMS. The mission of PCMS is to simplify integrating SMEs in a $\mathrm{VBE}$ and foster cooperation among SMEs members of the VBE by sharing their 
individual profiles of competencies, in order to achieve collaboratively new business opportunities (ECOLEAD D21.2a, 2005).

The PCMS could be considered as the knowledge base of companies belonging to the VBE. It contains enough information to find enterprises capable of participating in a business opportunity. Such information will be used for VO members to integrate their applications to business processes. Next section describes the architecture used to make application integration in a VBE context happen.

\section{PROCESS BASED INTEGRATION FRAMEWORK}

\subsection{Runtime Architecture}

Realizing process based integration requires the definition of an architecture which specifies components for process definition, a collaboration gateway, and enterprise's application linking. Figure 1 shows the architecture developed in order to enable seamless integration of application systems as services to business processes.
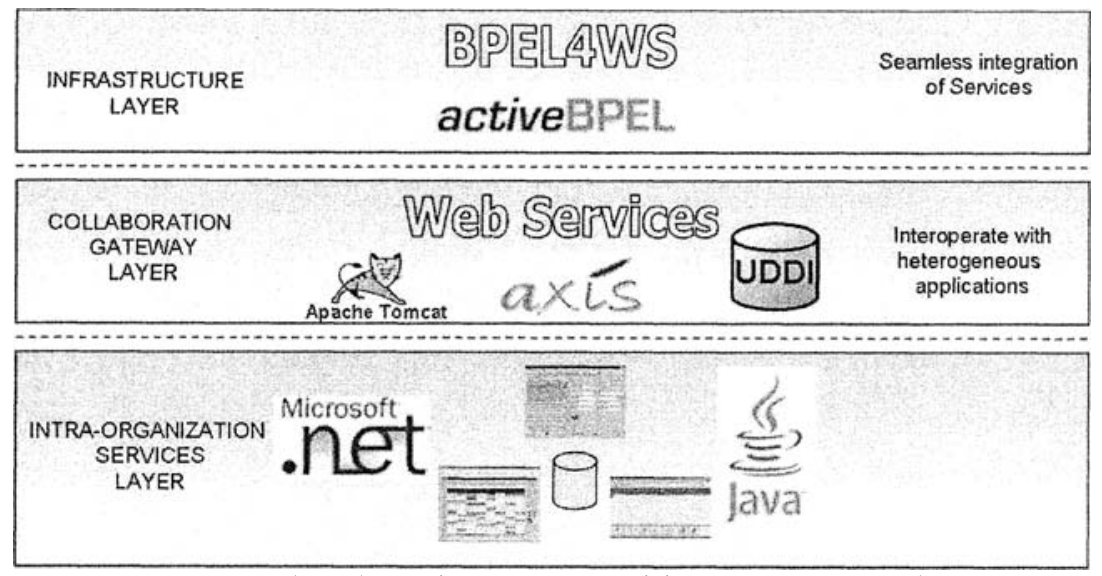

Figure 1. Process-based Architecture (adapted from ECOLEAD D61.2, 2005)

The architecture consists in three layers:

- Infrastructure layer. Manages business process execution; this layer enables users create, execute and keep track of business processes. The business process engine "activeBPEL" (www.activebpel.org) could be used for these tasks.

- Collaboration gateway layer. In this layer resides the Web services that allow information interchange between enterprise applications and the business processes, and it also contains the Web services registry for Universal Description, Discovery and Integration (UDDI) (www.uddi.org). A Web Service container for this layer could be "Axis" (www.apache.org/axis).

- Intra-organization services layer. The enterprises' applications are in this layer. Enterprises enable data access by creating Web services that behave 
as intermediaries to the applications, databases or end users and obtain the information needed.

The business process engine (activeBPEL) and the Web Service container (Axis) are supported by the Apache TomCat servlet container which resides in the Collaboration gateway layer. The following subsections show how integration is achieved using this architecture.

\subsection{Generic Business Processes}

A Generic Business Process (GBP) is a process template specifying a set of steps that define the business logic of operations among SMEs forming a VO. A GBP specification should:

- allows definition of business processes: order of execution of the constituent activities, the roles required to be played by partners, the messages exchange among these partners, and the fault and exception handling mechanisms;

- enables future integration with applications from different enterprises;

- provides an integration driver for communication between applications and processes using Web Services.

A modelling language that fits the requirements of a GBP specification is BPEL4WS (Business Process Execution Language for Web Services). It combines the features of a block structured process language (XLANG - Microsoft) with those of a graph-based process language (WSFL - IBM's).

A BPEL4WS process specification describes a flowchart. Each element in the process is an activity. Activities are connected through links to form directed acyclic graphs (Wohed, 2003). Three different types of files are necessary to describe a business process using BPEL4WS:

- a WSDL file for any Web service that will be used by the process;

- a Business Process (BPEL) file;

- a WSDL file for the process (i.e, the world will see the process as a web service).

The last point deserves special attention because it makes possible to integrate a process to other processes. Web Services are becoming a feature necessary to every programming language. A standard to orchestrate WSs, such as BPEL4WS facilitates the integration of applications developed with different programming languages and platforms. BPEL4WS is becoming a popular process definition language and as a consequence there are several open and proprietary process engines that implement the standard.

GBPs should be created to satisfy specific needs that VOs may have. GBP modelling is the responsibility of GBP providers. Figure 2 shows a very simple request for quotation process modelled using activeWebflow Professional (activeendpoints.com); the process flows as follow:

1. The process is instantiated when a request for quotation arrives, or in other words when the Web service that describes the process is invoked, it receives data about a product. 
2. The assign activity "Data_from_customer to_providers" translates the product data to a format that enterprises $\mathrm{A}$ and $\mathrm{B}$ can understand.

3. Activities "Quote_enterprise_A" and "Quote enterprise_B" invoke the corresponding Web services to get a quotation for the product.

4. The assign activity "Quotation info for assesment" transforms Quotations coming from Enterprise $\mathrm{A}$ and $\mathrm{B}$ to a format understandable by the assessment activity.

5. Activity "Make Quotation", invokes the assessor Web service and obtains the quotation ready to be sent to the customer.

6. "Reply_Quotation" delivers the quotation to the customer.

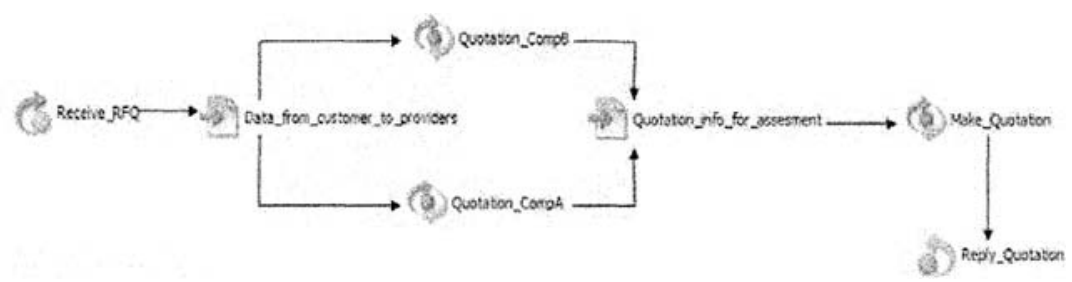

Figure 2. Request for quotation process

GBP providers have to test their processes within diverse scenarios, which imply GBP concretization, deployment and test as a SPB for every test scenario. SBPs are deployed on an ActiveBPEL server; this is performed by creating a BPR package which will contain: the BPEL process; the process deployment descriptor (PDD) which contains Web services endpoints and their relation to activities in the process; and a WSDL file for each Web Service used in the process (strongly recommended not necessary) including the WSDL for the process itself and the WSDL catalogue.

\subsection{A GBP Adaptation Tool}

A GBP is just a template whose parameters will be the real Web services necessary to interchange information with the applications systems. Once all parameters are concretized, a Specific Business Process (SBP) is produced. A SBP specifies concrete Web services therefore it is executable in a business process engine. Concretising SBPs from GBPs by hand could rapidly become a cumbersome task when multiple sources should be specified. This is roughly a mechanical task which uses information from the GBP definition, and from UDDI.

A software generator tool (GSPA - Generic to Specific Process Adaptor) was implemented assisting users to perform the necessary adaptations of GBPs to produce concrete SBPs, by guiding them in the specification necessary to concretize template processes. Figure 3 shows how GSPA works: first it analyzes GBPs looking for invoke activities that do not have a Web service associated. For every empty slot, it uses the UDDI to find out which Web services are available; then analyses the description files to resolve if they are compatible with the ones needed by the invoke activities.

Finally, GSPA integrates existing Web services to the target invoke activities by adding the endpoint URL and in case it is necessary, adds assign activities to 
transform data format from/to process/Web services. If Web services do not exist, the GSPA could create them along with Web forms (e.g., browser windows) to show the services input data and allow capturing the data that will be replied. The persons responsible of a VO can always decide in configuring the process manually, so they can choose which Web service will satisfy an invoke activity in a process and also set those particular configuration parameters needed by a process.

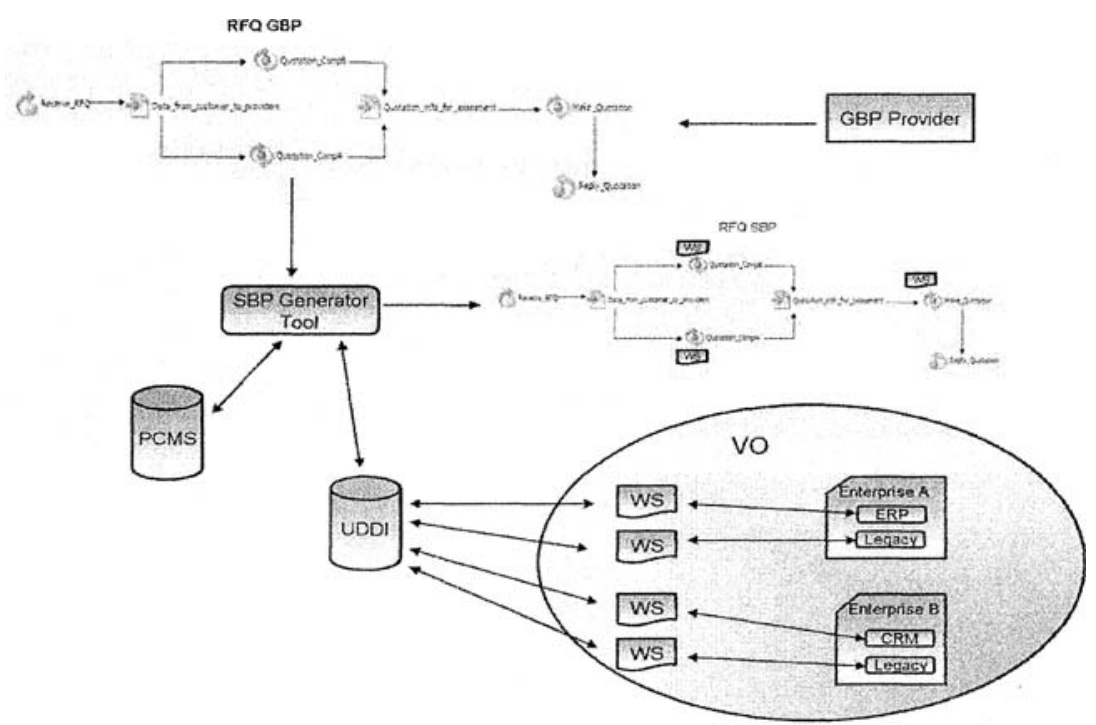

Figure 3. Specific Business Process generation

Using as example the Request for quotation process shown in Figure 2, and the diagram in Figure 3, the previous steps to create a SBP from a GBP could be described in detail as follows. The first user task is to provide GSPA with paths to the BPEL and PDD files. GSPA then will parse the PDD and find three partner links to be replaced. Then it goes to parse the BPEL to look for the invoke activities that use the partner links just found on the PDD, and can show the user which messages the process expect to send/receive to/from the Web service. The user will see a list of three invoke activities that require setting up Web services for each. Some details in the invoke activities, like the input and output messages, are gathered and shown to the user.

The second task a user should perform is to provide the companies names that will participate in the process and their Web Services information. GSPA will search for Web Services belonging to the companies and match them with the ones needed by the process. The user will finally decide the matching between invoke activities and the companies' Web services; but GSPA will make easier these tasks by performing a business process context search in the UDDI and parsing those companies' Web services that are associated. Then GSPA compares the invoke activities messages against the Web services messages to find out whether they are compatible, and finally suggests the instantiation. 
The third user task is to detail the SBP. GSPA shows the matching suggestions and also the services that didn't match but are available. The user can decide whether the selected services satisfy the particular requirement. The user can also make changes. For instance, do not use the suggestions and select other Web services from the ones available, or in the worst case, provide the address location of the Web service she prefers to use. After all invoke activities have an associated Web service, the SBP will be ready for deployment.

The final user task is to generate the BPR package for deployment. To do so, the user just has to let GSPA know the activeBpel server location where the package will be deployed. GSPA will generate a WSDL file for each Web service used in the process, make the necessary changes to BPEL and PDD files to work with the selected Web services, create the WSDL catalogue, create the BPR package and deploy it to the server specified. A customer can start the deployed process by invoking it as a Web service.

In the described way GSPA makes SBP concretization a straightforward four step process (for the user) and also makes it look almost like a plug and play process. Figure 4 shows the SBP generation process in detail, with the roles that every participants play.

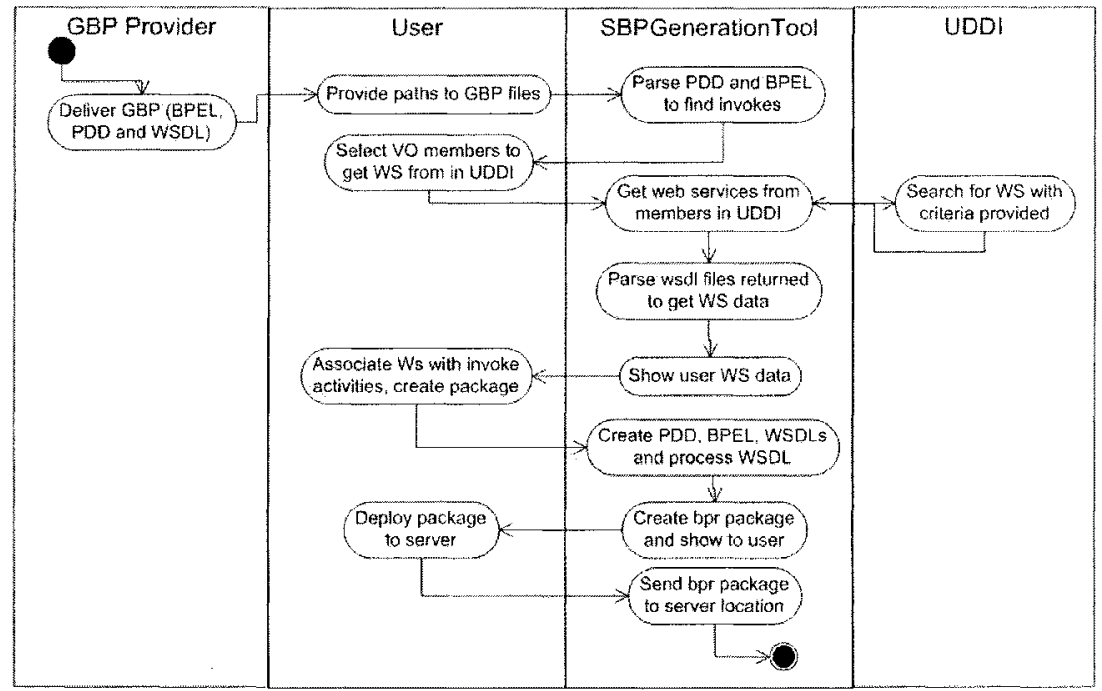

Figure 4. SBP generation process

As a corollary to the approach described, several things can be noticed. First of all, each VO partner can focus on their business problems without concerning other partners. A GBP provider worries on how to provide good-quality business processes to their users, with the best practices and as generic as possible, so those processes satisfy user needs when participating in VOs. The GBP includes in its specification a way to communicate with it so an enterprise can generate compatible Web services. 
On the other hand, enterprises forming part of a VO enable access to their applications by creating Web services compatible with Business Process of interest. The SBP generator tool makes easier to particularize a GBP to a certain VO. As long as the GBP provider gives a correct process and the information from the $\mathrm{VO}$ is available, the GSPA is able to concretize a SBP from a GBP.

GSPA was designed with simplicity in mind, thus the SBP Generation Process is a simple one. One goal was make the user interface intuitive and following the process shown in Figure 4 and described above. Figure 5 shows the first screen of GSPA.

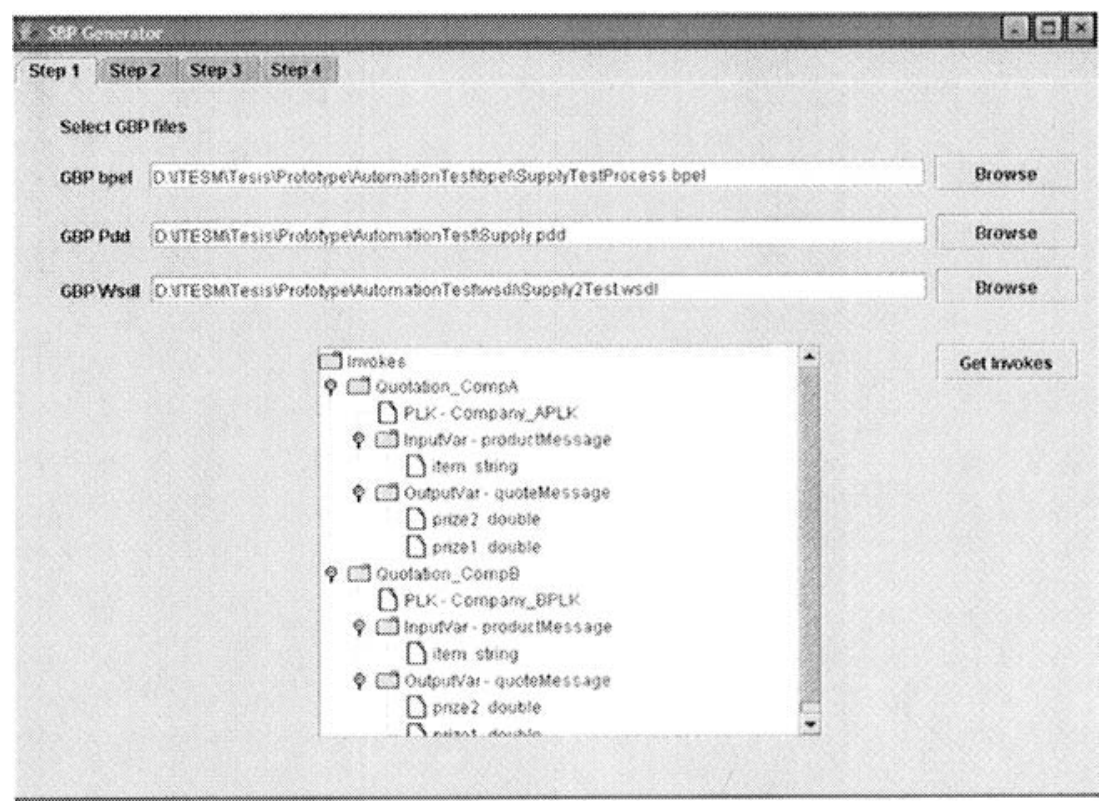

Figure 5 SBP Generator Tool - Step l

Using GSPA the user will be able to do the following tasks:

- Step1 tab. Load GBP bpel, pdd and wsdl, and analyse the GBP in order to find which invoke activities need a Web Service associated.

- Step2 tab. Search the UDDI for Web Services belonging to VO members.

- Step3 tab. Associate the invoke activities specified in Step 1 tab with the Web Services found in Step 2; and generate the SBP deployment package. See Figure 6.

- Step4 tab. Finally on this tab the user can deploy the SBP to the server.

The GBP that is being instantiated in Figure 5 and 6 is the Request for quotation process which was shown in Figure 2 and previously described.

Another interesting feature of GSPA is that if the user needs to generate several SBPs from one GBP, she just needs to go to the Step2 tab, change the invoke activities - Web Services associations and deploy the process in the Step4 tab. 
GSPA is already functional but still needs some improvements. For instance, very important is a simpler approach to provide users with a more consistent invoke activity - web service suggest association.

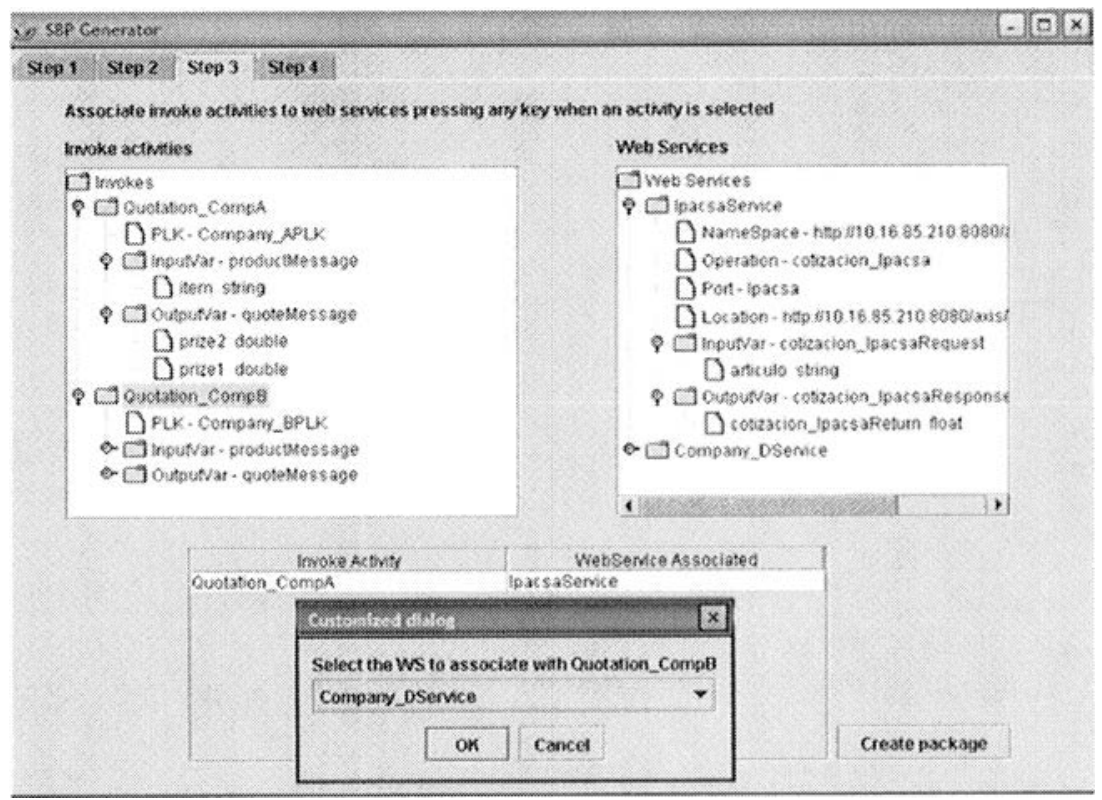

Figure 5 SBP Generator Tool - Step3

GSPA has been tested in processes more complex than the one described here, one of them is a Production Sales Forecast Process. This process involves several SBPs from two GBPs: the Production Forecast Process and the Sales Forecast Process. In this last experiment we enjoyed the versatility of BPEL. It was straightforward to compose processes from existing ones, which gave us a big spectrum for integration possibilities.

There is more to a VBE than business processes concretization. Many issues need to be solved for the VBE to be effective as a means to promote the dynamic creation of VOs. Different legal, sociological and technical aspects need to be set out. However, technical propositions are necessary to understand the advantages that a VBE should provide in order to be more effective. The research presented in this paper is an example of how information in a VBE could be used to automate VO creation. Still more research needs to be conducted to solve other problems faced at VBE and VO management.

\section{CONCLUSIONS AND FURTHER WORK}

Virtual Breeding Environments constitute an ecosystem necessary for creating and increasing trust among enterprises thus they are able to collaborate on networks of 
partners offering goods or services. However, VBEs could be more useful if extended with tools to simplify dynamic VO creation.

This paper showed how the information stored in a VBE could be augmented including additional details about applications that member enterprises export as services and templates of processes describing the operation of a VO. Using such information, an integration tool was developed to produce concrete processes necessary to manage a VO's operation.

The research reported cover just part of the whole problem for dynamic VO creation and management. There are several other necessary functions to manage the operation of a VO and decide what information has to be recorded at the time when a VO is dissolved. These requirements will be part of future research we need to conduct.

\section{ACKNOWLEDGMENTS}

This work was supported by the ECOLEAD (European Collaborative Networked Organisations LEADership initiative) project and the Instituto Tecnologico y de Estudios Superiores de Monterrey (ITESM), Campus Monterrey. The authors thank the contributions of ECOLEAD partners. Also, the authors thank Active Endpoints and the Microsoft Research Lab at ITESM, who provided us academic licences of ActiveBpel Designer and Microsoft UDDI servers, respectively.

\section{REFERENCES}

1. Camarinha-Matos, L. VOSTER Report on Infrastructures and Interoperability for VOs. VOSTER. Deliverable D44. 2003

2. Camarinha-Matos, L. Afarmanesh, H. Collaborative Networked Organizations. A research agenda for emerging business models, pages 5-8. USA: Springer. 2004

3. Chadwick, D., Dimitrakos, T., Kleese-Van Dam, K., Damian, R., Matthews, B., Otenko, A. Multilayer Privilege Management for Dynamic Collaborative Scientific Communities, UK Workshop on Grid Security Experiences, July 2004.

4. ECOLEAD internal document deliverable D21.1, Edited by Afarmanesh, H. Galeano, N. CamarinhaMatos, L. D21.1 Characterization of Key Components, Features, and Operating Principles of the Virtual Breeding Environment. 2005

5. ECOLEAD internal document deliverable D21.2a, Edited by Ermilova, E. Galeano, N. Afsarmanesh, H. D21.2a Specification of the VBE competency/profile management . 2005

6. ECOLEAD internal document deliverable D61.2, Edited by Arana, C., Ma del Mar, R. Global approach for a horizontal infrastructure architecture. 2005

7. Hass, H. Brown, A. Web Services glossary. Retrieved November 17, 2005, from http:/Www.w3.org/TR/ws-gloss/\#defs. 2004

8. Nayak, N., Bhaskaran, K., \& Das, zR. Virtual enterprises-building blocks for dynamic e-business. Information Technology for Virtual Enterprises, 2001; 29-30, 80-87.

9. Wohed, P., Van Der Aalst, W.M.P., Dumas, M., Hofstede, A.H.M. Analysis of Web Services Composition Languages: The Case of BPEL4WS. 22nd International Conference on Conceptual Modeling (ER 2003), volume 2813 of Lecture Notes in Computer Science, pages 200-215. Berlin: Springer-Verlag. 2003. 